\title{
AJUSTE, SELEÇÃO E TESTE DE IDENTIDADE DE MODELO PARA VOLUME E NÚMERO DE MOIRÕES DA CANDEIA (Eremanthus erythropappus)
}

\author{
José Fabio Camolesi ${ }^{1}$, José Roberto Soares Scolforo ${ }^{2}$, Antonio Donizette de Oliveira ${ }^{3}$, \\ Fausto Weimar Acerbi Júnior ${ }^{4}$, Ana Luiza Rufini ${ }^{5}$, José Márcio de Mello ${ }^{6}$
}

(recebido: 11 de setembro de 2009; aceito: 29 de julho de 2010)

\begin{abstract}
RESUMO: Conduziu-se este estudo, com os objetivos de ajustar e selecionar modelos estatísticos para a estimativa do volume total, com e sem casca, e o número de moirões, em três municípios de Minas Gerais; definir o fator de empilhamento por classe de diâmetro para a espécie e avaliar a possibilidade de agrupamento dos modelos estatísticos com base no teste de identidade de modelos. A base de dados foi composta de 174 árvores distribuídas em seis classes de diâmetro, nas três regiões de estudo, as quais foram cubadas usando um xilômetro. A acurácia dos modelos foi avaliada por meio do coeficiente de determinação, desvio padrão da média e análise gráfica dos resíduos. Concluiu-se que o fator de empilhamento decresce com o aumento das classes diamétricas. O modelo selecionado para todas as variáveis testadas foi o de Spurr logaritmizado. O teste de identidade entre modelos indicou a possibilidade de agrupamento entre os municípios de Aiuruoca e Ouro Preto para as variáveis volume total com casca e volume total sem casca, enquanto que para a variável número de moirões esse teste indicou não ser possível o agrupamento de nenhuma combinação entre os municípios estudados.
\end{abstract}

Palavras-chave: Fator de empilhamento, cubagem rigorosa, xilômetro.

\section{FITTING, SELECTION AND MODEL IDENTITY TEST FOR VOLUME AND NUMBER OF FENCE POSTS OF CANDEIA (Eremanthus erythropappus)}

\begin{abstract}
The objectives of this study were: to fit and select statistical models for estimating the total volume, with and without bark, and number of fence posts in three counties of Minas Gerais State, as well as, to define the stack factor per diameter class and to evaluate, by means of the identity model test, the possibility of using the same mathematical model for all regions. Data base were obtained from a scaling of 174 trees grouped in six diameter classes within the three study regions. The scaling was carried out using the xylometer method (water displacement technique). The accuracy of the models was evaluated using the coefficient of determination, the mean standard error of estimate, and the graphical analysis of residuals. The Spurr logarithm model was selected as the best one for all tested variables. Considering the variables total volume, with and without bark, the identity test showed the possibility of using the same model for Aiuruoca and Ouro Preto counties whereas for the variable number of fence posts, the identity test showed that there is no possibility of grouping for any combination among the counties.
\end{abstract}

Key words: Stack factor, scaling, xylometer.

\section{INTRODUÇÃO}

O desenvolvimento econômico e social de qualquer país está intimamente ligado aos seus recursos naturais. Diversas atividades das indústrias dos setores farmacêutico, cosmético, alimentício e uma variada gama de utilidades imprescindíveis ao bem-estar dos homens têm sua origem em produtos florestais.
Segundo Carvalho (1994), as regiões de cerrado se apresentam como um importante bioma para a obtenção de produtos fitoquímicos de grande valor comercial. Dentre muitas essências de valor econômico, destaca-se a candeia (Eremanthus erythropappus), uma espécie arbórea pertencente à família das Asteraceae que, frequentemente, é encontrada em áreas montano-campestres nos estados de Minas Gerais, Bahia, Espírito Santo e Rio de Janeiro.

${ }^{1}$ Engenheiro Florestal, Mestre em Engenharia Florestal - Departamento de Ciências Florestais - Universidade Federal de Lavras - Cx. P. 3037 - 37200-000 - Lavras, MG - camolesi@uflanet.com.br

${ }^{2}$ Engenheiro Florestal, Professor Doutor em Engenharia Florestal - Departamento de Ciências Florestais - Universidade Federal de Lavras - Cx. P. 3037 - 37200-000 - Lavras, MG - scolforo@dcf.ufla.br

${ }^{3}$ Engenheiro Florestal, Professor Doutor em Ciência Florestal - Departamento de Ciências Florestais - Universidade Federal de Lavras - Cx. P. 3037 - 37200-000 - Lavras, MG - donizete@ dcf.ufla.br

${ }^{4}$ Engenheiro Florestal, Professor Doutorando em Sensoriamento Remoto - Departamento de Ciências Florestais - Universidade Federal de Lavras - Cx. P. 3037 - 37200-000 - Lavras, MG - fausto@ dcf.ufla.br

${ }^{5}$ Engenheira Florestal, Doutoranda em Engenharia Florestal - Programa de Pós-Graduação em Engenharia Florestal - Departamento de Ciências Florestais - Universidade Federal de Lavras - Cx. P. 3037 - 37200-000 - Lavras, MG - alrufini@ dcf.ufla.br ${ }^{6}$ Engenheiro Florestal, Professor Doutor em Recursos Florestais - Departamento de Ciências Florestais - Universidade Federal de Lavras Cx. P. 3037 - 37200-000 - Lavras, MG - josemarcio@dcf.ufla.br 
A candeia possui madeira resistente ao ataque de cupins e bactérias, sendo por isso muito utilizada como moirões, esteios, caibros, postes, tacos, dormentes e vigas. O óleo essencial extraído da madeira tem diversas aplicações nas indústrias de cosméticos e fármacos. Decorrente dessas propriedades interessantes, existe forte pressão para o corte dessa espécie. Assim, são necessários estudos para balizarem a elaboração de planos de manejo sustentáveis para a mesma, a fim de evitar a sua exploração de maneira predatória e a sua exaustão ou desaparecimento.

Em estudos realizados no município de Aiuruoca-MG, Pérez (2001) concluiu que a equação de volume de Schummacher e Hall na forma logarítmica teve o melhor desempenho, dentre os modelos avaliados, para a estimativa do volume e do número de moirões para a candeia. Entretanto, deve-se considerar que a região de ocorrência da candeia abrange 457 municípios do estado de Minas Gerais, o que corresponde a $34,38 \%$ do estado. Assim, o uso genérico desse modelo para qualquer um desses municípios, sem um estudo prévio, é uma ação que não é factível com um plano de manejo sustentado. Dessa forma, é de fundamental importância realizar estudos visando a comparar modelos entre as diferentes regiões.

Dada a importância econômica e ambiental da candeia, o uso da modelagem matemática para estimativa de volume e número de moirões é ponto fundamental para se estabelecer um bom plano de manejo. Assim, neste trabalho, objetivou-se ajustar e selecionar modelos estatísticos para a estimativa do volume total, com e sem casca, e o número de moirões, em três municípios de Minas Gerais; definir o fator de empilhamento por classe de diâmetro para a espécie e avaliar a possibilidade de agrupamento dos modelos estatísticos com base no teste de identidade de modelos.

\section{MATERIAL E MÉTODOS}

\subsection{Regiões de estudo}

A área de ocorrência da candeia (Eremanthus erythropappus), no estado de Minas Gerais e os três municípios em que foram obtidos os dados para a realização deste estudo são mostrados na Figura 1. Na Tabela 1, são apresentadas informações sobre latitude, longitude e altitude média das áreas onde foram coletados os dados.
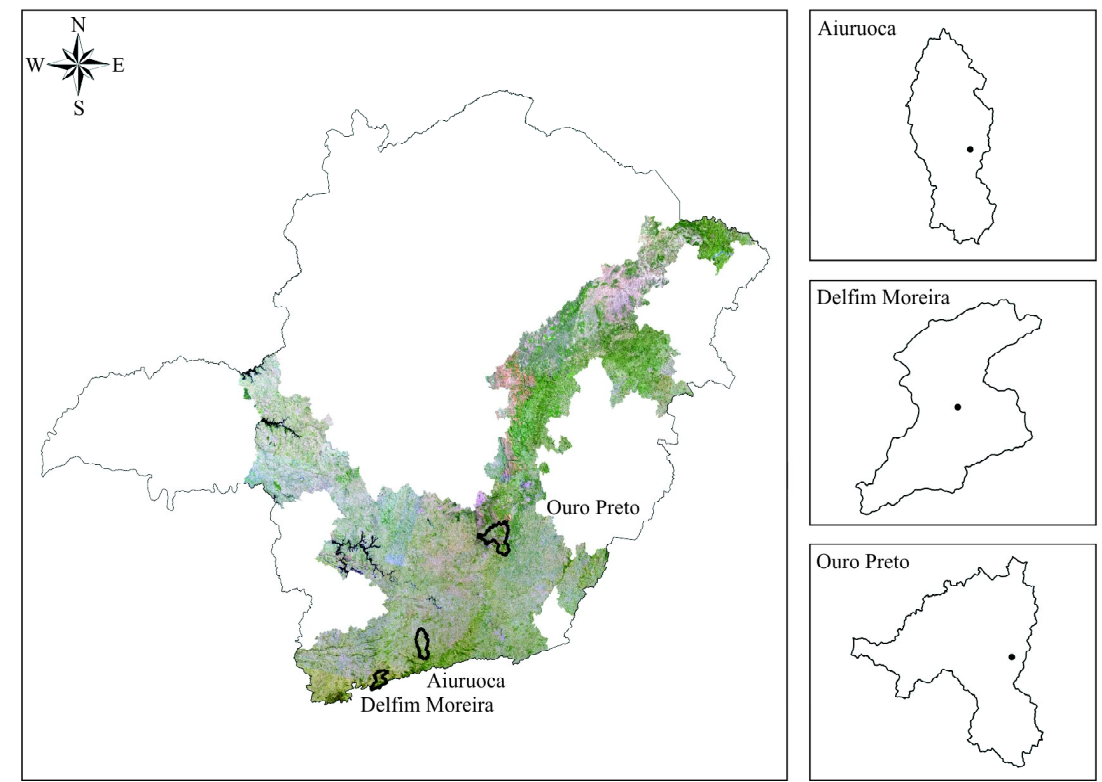

Figura 1 - Distribuição espacial da área de ocorrência da candeia no estado de Minas Gerais e a localização dos municípios onde foram coletados os dados.

Figure 1 - Spatial distribution of candeia trees in the state of Minas Gerais and localization of the three counties where the data were collected.

Cerne, Lavras, v. 16, n. 4, p. 431-441, out./dez. 2010 
Tabela 1 - Informações sobre latitude, longitude e altitude média das áreas onde foram coletados os dados.

Table 1 - Latitude, longitude and mean altitude of the study area.

\begin{tabular}{lccc}
\hline Município & Latitude & Longitude & Altitude \\
\hline Delfim Moreira & $23^{\circ} 25^{\prime}$ & $45^{\circ} 15^{\prime}$ & $1600 \mathrm{~m}$ \\
Aiuruoca & $21^{\circ} 56^{\prime}$ & $44^{\circ} 38^{\prime}$ & $1572 \mathrm{~m}$ \\
Ouro Preto & $20^{\circ} 23^{\prime}$ & $43^{\circ} 33^{\prime}$ & $1545 \mathrm{~m}$ \\
\hline
\end{tabular}

\subsection{Amostragem e coleta dos dados}

Para a cubagem rigorosa das árvores seguiu-se o esquema mostrado na Figura 2. Antes de derrubar a árvore, foram medidos o diâmetro à altura do peito e a espessura da casca nesse ponto. Após a derrubada da árvore, mediram-se as alturas total e comercial, sendo a referência da altura comercial o comprimento da base até o galho mais extenso, tendo como diâmetro mínimo $3 \mathrm{~cm}$.

Contabilizou-se o número de moirões de $2,2 \mathrm{~m}$ de comprimento, com $10 \mathrm{~cm}$ de diâmetro, na metade do moirão, ou 7,5 cm na extremidade mais fina possível de serem extraídos da árvore. Para as árvores das classes de diâmetro cujo valor central é de 7,5, 12,5 e 17,5 cm, os moirões foram contabilizados como moirões roliços. Para a classe cujo valor central é 22,5 , os moirões do fuste foram contabilizados como partidos (lascados), sendo a peça dividida em dois moirões, quando possível. Para a classe de diâmetro de valor central $27,5 \mathrm{~cm}$, os moirões do fuste foram contabilizados como lascados, sendo a peça dividida de duas a quatro partes, conforme as possibilidades das peças. Para a classe de diâmetro de valor central $32,5 \mathrm{~cm}$, os moirões do fuste foram contabilizados como lascados, sendo a peça dividida de três a seis partes, de acordo com as possibilidades da peça.

Procurou-se contabilizar o maior número de moirões que uma árvore podia fornecer. Porém, quando ocorre exploração, o proprietário, sempre que possível, reserva peças de maior diâmetro e mais retilíneas, com comprimentos que vão de 3,5 a $6 \mathrm{~m}$ de comprimento. Essas peças alcançam valores mais altos e são destinadas à construção de currais, celeiros e barracões.

Após as medições, seccionou-se a árvore nas posições correspondentes a $0 \%, 25 \%, 50 \%, 75 \%$ e $100 \%$ da altura comercial, resultando em quatro seções. Em cada seção retirou-se um disco para as análises especificadas a seguir.

\subsection{Espessura de casca}

Mediu-se a espessura da casca de cada disco retirado nas seções, os quais foram utilizados para obter a porcentagem de casca e o volume de casca da árvore dentro de cada classe de diâmetro. Para isso, foram utilizadas as expressões a seguir (SCOLFORO \& THIERSCH, 2004).

$V_{c \%}=\left[1-\left(\frac{d}{D}\right)^{2}\right] * 100$

em que $V_{c \%}$ é o volume de casca, em porcentagem; $d$ é o diâmetro a altura do peito sem casca, em $\mathrm{cm}$; e $D$ é o diâmetro à altura do peito com casca, em $\mathrm{cm}$.

$V_{c}=V_{c / c}-V_{s / c}$

em que $V_{c}$ é o volume de casca, em $\mathrm{m}^{3} ; V_{c / c}$ é o volume com casca, em $\mathrm{m}^{3} ;$ e $V_{s / c}$ é o volume sem casca, em $\mathrm{m}^{3}$.

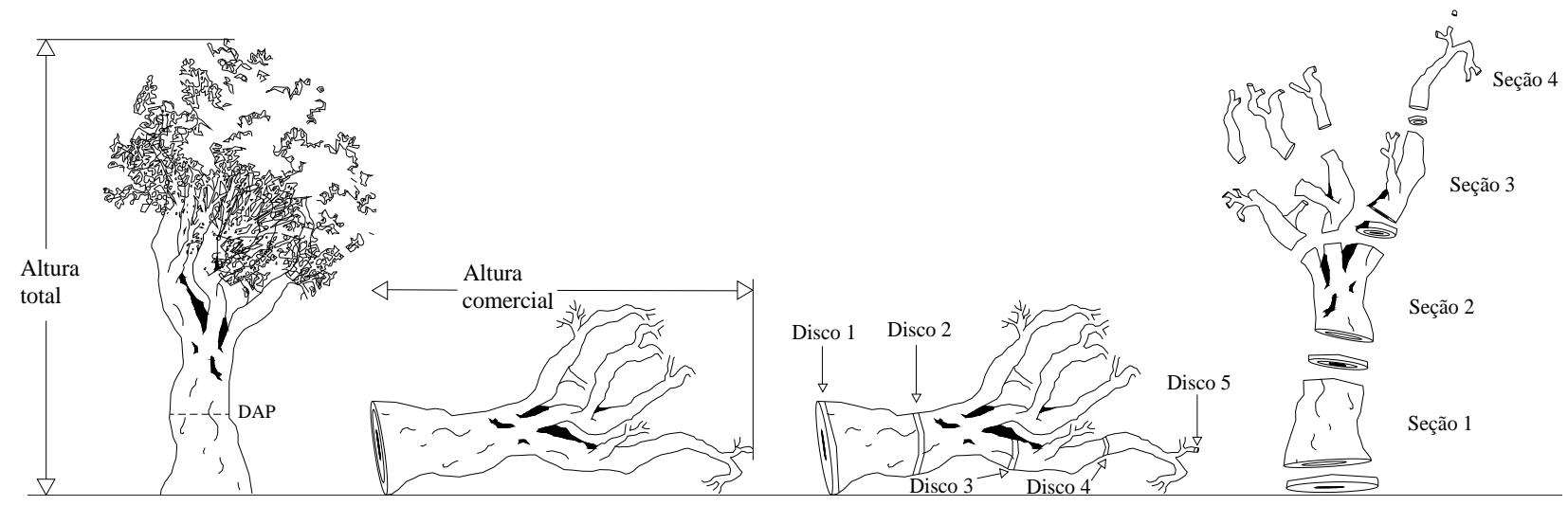

Figura 2 - Esquema de coleta de dados nas árvores cubadas.

Figure 2 - Data collection scheme in the scaling trees 


\subsection{Quantificação do volume real}

Para determinar o volume real de madeira da árvore até $3 \mathrm{~cm}$ de diâmetro com casca, exclusive o toco, utilizou-se o xilômetro. Esse equipamento foi calibrado de forma que o volume de dois litros de água $\left(0,002 \mathrm{~m}^{3}\right)$, equivale a um deslocamento do menisco de $0,84167 \mathrm{~cm}$. Esses valores foram utilizados como constantes na obtenção do volume real em metros cúbicos para cada árvore. A expressão a seguir foi elaborada para esta finalidade.

$V t=\left(\frac{D s_{1} * K}{W}\right)+\left(\frac{D s_{2} * K}{W}\right)+\left(\frac{D s_{3} * K}{W}\right)+\left(\frac{D s_{4} * K}{W}\right)+\left(\frac{D d_{i} * K}{W}\right)$

em que $D s_{i}$ é o deslocamento de água pela madeira de uma sessão, em cm; $K$ e $W$ são constantes do equipamento para conversão do deslocamento de água, em $\mathrm{cm}$, para volume, em m $\left(K=0,002 \mathrm{~m}^{3}\right.$ e $\left.W=0,84167 \mathrm{~cm}\right)$; e $D d_{i}$ é o deslocamento de água dos discos de cada árvore.

Para cada árvore cubada rigorosamente, mediramse a altura e a circunferência do toco, sendo esta tomada na extremidade superior do toco. Com estas medidas, calculou-se o volume do toco, considerando-o como sendo o volume de um cilindro, utilizando a seguinte expressão:

$V_{\text {toco }}=\frac{\pi^{*} d^{2}}{40000} * l$

em que $V_{\text {toco }}$ é o volume do toco, em $\mathrm{m}^{3}$; $d$ é o diâmetro, tomado na extremidade superior do toco, em $\mathrm{cm}$; e $l$ é o comprimento do toco, $\mathrm{m}$.

Para obter o volume de madeira empilhada, as árvores cubadas rigorosamente foram empilhadas, mantendo-se o controle por classe de diâmetro. Em cada pilha de madeira mediram-se a altura, a largura e o comprimento em vários pontos, que variaram de acordo com o comprimento da pilha de madeira. A partir do produto das medidas de altura, largura e comprimento de cada pilha, dentro de cada classe, obteve-se o volume da pilha.

Com base nos volumes reais obtidos por meio do xilômetro, obteve-se o fator de empilhamento por classe de diâmetro e para todo o conjunto de árvores. $\mathrm{O}$ fator de empilhamento é a razão entre o volume empilhado e o volume real, sendo sempre superior a 1 e permite converter o volume sólido $\left(\mathrm{m}^{3}\right)$ para volume em metros estéreo.

Foram amostradas 174 árvores distribuídas em 6 classes de diâmetro, com diâmetro mínimo de $5 \mathrm{~cm}$ e máximo de $35 \mathrm{~cm}$ (Tabela 2).
Tabela 2 - Número de árvores cubadas nas áreas de estudo, por classe de diâmetro.

Table 2 - Frequency of trees scaled, per diameter class, in the study areas.

\begin{tabular}{cccc}
\hline \multirow{2}{*}{$\begin{array}{c}\text { Classes de } \\
\text { diâmetro }\end{array}$} & \multicolumn{3}{c}{ Frequência } \\
\cline { 2 - 4 } & Delfim Moreira & Aiuruoca & Ouro Preto \\
\hline $5 \rightarrow 10$ & 10 & 10 & 10 \\
$10 \rightarrow 15$ & 11 & 10 & 11 \\
$15 \rightarrow 20$ & 10 & 10 & 11 \\
$20 \rightarrow 25$ & 10 & 10 & 10 \\
$25 \rightarrow 30$ & 7 & 10 & 10 \\
$30 \rightarrow 35$ & 4 & 10 & 10 \\
\hline
\end{tabular}

\subsection{Modelos para estimar o volume de madeira e a quantidade de moirões}

Foram ajustados doze modelos considerando as variáveis dependentes volume total com casca, volume total sem casca e número de moirões, em função de variáveis dendrométricas (DAP e H) (Tabela 3). Para selecionar os modelos, levou-se em consideração o coeficiente de determinação ajustado, o erro padrão residual corrigido em porcentagem e a análise gráfica dos resíduos.

\subsection{Teste de identidade entre modelos}

Realizou-se o teste de identidade entre modelos descrito por Graybill (1976) com o propósito de avaliar a possibilidade de uma única equação representar o comportamento das variáveis estudadas. Para cada variável, foram testadas todas as combinações que representam as várias possibilidades de agrupamento entre as três regiões estudadas. A identificação da igualdade ou da diferença entre as regiões, em relação às variáveis modeladas, foi feita por meio da comparação entre a soma de quadrado dos resíduos para cada variável, em cada região (modelo completo) e a soma de quadrado da diferença para o modelo ajustado com uma única base de dados contendo todas as informações das três regiões de estudo (modelo reduzido). Sempre que o valor de F calculado for maior ou igual ao valor de F tabelado, significa que o teste foi significativo, a um determinado nível de probabilidade $(\alpha)$ pré-estabelecido. Nesse caso, rejeita-se $\mathrm{H}_{0}$, ou seja, o total da soma de quadrados dos resíduos de cada uma das variáveis em cada região (modelo completo) difere, estatisticamente, da soma de

Cerne, Lavras, v. 16, n. 4, p. 431-441, out./dez. 2010 
Tabela 3 - Modelos ajustados para volume de madeira e número de moirões.

Table 3 - Fitted models for wood volume and number of fence posts.

\begin{tabular}{|c|c|}
\hline Autor & Modelo \\
\hline Hohenald-Krenm & $Y=\beta_{0}+\beta_{1} \operatorname{Dap}+\beta_{2}\left(\operatorname{Dap}^{2}\right)+\varepsilon_{i}$ \\
\hline Brenac & $\operatorname{Ln}(Y)=\beta_{0}+\beta_{1} \operatorname{Ln}(\operatorname{Dap})+\beta_{2}\left(\frac{1}{\operatorname{Dap}}\right)+\operatorname{Ln}\left(\varepsilon_{i}\right)$ \\
\hline Spurr & $Y=\beta_{0}+\beta_{1}\left(\operatorname{Dap}^{2} H\right)+\varepsilon_{i}$ \\
\hline Schummacher-Hall & $Y=\beta_{0} * \operatorname{Dap}^{\beta_{1}} * H^{\beta_{2}}+\varepsilon_{i}$ \\
\hline Schummacher-Hall (logarítmica) & $\operatorname{Ln}(Y)=\beta_{0}+\beta_{1} \operatorname{Ln}(\operatorname{Dap})+\beta_{2} \operatorname{Ln}(H)+\operatorname{Ln} \varepsilon_{i}$ \\
\hline Honner & $Y=\frac{\operatorname{Dap}^{2}}{\beta_{0}+\beta_{1}\left(\frac{1}{H}\right)}+\varepsilon_{i}$ \\
\hline Ogaya & $Y=\operatorname{Dap}^{2}\left(\beta_{0}+\beta_{1} H\right)+\varepsilon_{i}$ \\
\hline Stoat (australiana) & $Y=\beta_{0}+\beta_{1} \operatorname{Dap}^{2}+\beta_{2}\left(\operatorname{Dap}^{2} H\right)+\beta_{3} H+\varepsilon_{i}$ \\
\hline Naslund & $Y=\beta_{1} \operatorname{Dap}^{2}+\beta_{2}\left(\operatorname{Dap}^{2} H\right)+\beta_{3}\left(D a p H^{2}\right)+\beta_{4} H^{2}+\varepsilon_{i}$ \\
\hline Takata & $Y=\frac{\left(\operatorname{Dap}^{2} H\right)}{\left(\beta_{0}+\beta_{1} \text { Dap }\right)}+\varepsilon_{i}$ \\
\hline Spurr (logarítmica) & $\operatorname{Ln}(Y)=\beta_{0}+\beta_{1} \operatorname{Ln}\left(\operatorname{Dap}^{2} H\right)+\operatorname{Ln} \varepsilon_{i}$ \\
\hline Meyer & $V=\beta_{0}+\beta_{1} D a p+\beta_{2} D a p^{2}+\beta_{3} D a p H+\beta_{4} D a p^{2} H+\beta_{5} H+\varepsilon_{i}$ \\
\hline
\end{tabular}

$\beta_{i}$ - parâmetros da equação; Dap - diâmetro à altura do peito; $H$ - altura; $Y$ - característica de interesse (volumes e número de moirões); $L n$ - logaritmo neperiano e $\varepsilon_{i}$ - erro de estimativa.

Fonte: (SCOLFORO, 2005).

quadrado da diferença para o modelo ajustado para o conjunto total dos dados para cada variável (modelo reduzido). Caso contrário, se aceita $\mathrm{H}_{0}$, ou seja, pode-se utilizar um único modelo para estimar a variável estudada nas três regiões.

\section{RESULTADOS E DISCUSSÕES}

\subsection{Precisão e acurácia dos modelos ajustados para estimativa de volumes e número de moirões}

As estimativas dos parâmetros dos modelos ajustados e selecionados e as medidas de precisão das equações estão sumarizadas na Tabela 4. Os valores de coeficiente de determinação das equações volumétricas e de número de moirões situaram-se próximos a $98 \%$ e $90 \%$, respectivamente. $\mathrm{O}$ erro padrão da estimativa expresso em porcentagem variou de $18,62 \%$ a $26,28 \%$ para as equações volumétricas e de $29,43 \%$ a $33,54 \%$ para as equações de número de moirões.
Na distribuição gráfica dos resíduos (Figura 3), nota-se que não há tendenciosidade. O erro de estimativa para a variável volume de um único indivíduo apresentase elevado, e mostra que os erros de superestimativa estão anulando os erros de subestimativa. Para a variável número de moirões, a distribuição gráfica dos resíduos apresenta uma pequena heterogeneidade. De acordo com resultados obtidos por Pérez (2001), trabalhando com a mesma espécie de candeia no município de Aiuruoca, os comportamentos apresentados pelas estimativas dos parâmetros garantem um bom uso das equações, quando aplicadas em povoamentos nos três municípios de estudo, sendo este o objetivo que se buscou atingir.

De acordo com a avaliação dos doze modelos testados por meio do coeficiente de determinação $\left(\mathrm{R}^{2}{ }_{\text {ajst }}\right)$, erro padrão da estimativa (Syx) e análise da distribuição gráfica de resíduos, o modelo de Spurr logarítmico mostrou ser o mais adequado.

Cerne, Lavras, v. 16, n. 4, p. 431-441, out./dez. 2010 
Tabela 4 - Estimativas dos parâmetros e medidas de precisão das equações de volumes e número de moirões.

Table 4-Parameters estimated and precision measures for volume and number of fence posts equations.

\begin{tabular}{|c|c|c|c|c|c|}
\hline \multirow[t]{2}{*}{ Região } & \multicolumn{5}{|l|}{ Volume total com casca } \\
\hline & Equações de melhor ajuste & $\mathrm{F}_{\mathrm{cal}}$ & $\mathrm{R}^{2}(\%)$ & Syx $\left(\mathrm{m}^{3}\right)$ & Syx \% \\
\hline $\begin{array}{l}\text { Delfim } \\
\text { Moreira }\end{array}$ & $\operatorname{Ln}(\mathrm{VT})=-10,0695707796+1,002283589 * \operatorname{Ln}\left(\left(\mathrm{DAP}^{2}\right) * \mathrm{HT}\right)$ & 3238,21 & 98,45 & $\pm 0,0308$ & 19,97 \\
\hline Aiuruoca & $\operatorname{Ln}(\mathrm{VT})=-10,1434754209+1,0147112123 * \operatorname{Ln}\left(\left(\mathrm{DAP}^{2}\right) * \mathrm{HT}\right)$ & 3137,04 & 98,15 & $\pm 0,0503$ & 23,36 \\
\hline \multirow[t]{3}{*}{ Ouro Preto } & $\operatorname{Ln}(\mathrm{VT})=-9,9898114646+1,0016476429 * \operatorname{Ln}\left(\left(\mathrm{DAP}^{2}\right) * \mathrm{HT}\right)$ & 3809,94 & 98,42 & $\pm 0,0621$ & 26,28 \\
\hline & \multicolumn{5}{|l|}{ Volume total sem casca } \\
\hline & Equações de melhor ajuste & $\mathrm{F}_{\mathrm{cal}}$ & $\mathrm{R}^{2}(\%)$ & Syx $\left(\mathrm{m}^{3}\right)$ & Syx \% \\
\hline $\begin{array}{l}\text { Delfim } \\
\text { Moreira }\end{array}$ & $\operatorname{Ln}(\mathrm{VTsc})=-10,471757251+1,0284915738 * \operatorname{Ln}\left(\left(\mathrm{DAP}^{2}\right) * \mathrm{HT}\right)$ & 3583,69 & 98,60 & $\pm 0,0240$ & 18,62 \\
\hline Aiuruoca & $\operatorname{Ln}(\mathrm{VTsc})=-10,640696676+1,0501625078 * \operatorname{Ln}\left(\left(\mathrm{DAP}^{2}\right) * \mathrm{HT}\right)$ & 3271,25 & 98,23 & $\pm 0,0445$ & 24,72 \\
\hline \multirow[t]{3}{*}{ Ouro Preto } & $\operatorname{Ln}(\mathrm{VTsc})=-10,4573447531+1,0384037531 * \operatorname{Ln}\left(\left(\mathrm{DAP}^{2}\right) * \mathrm{HT}\right)$ & 4141,97 & 98,55 & $\pm 0,0531$ & 25,91 \\
\hline & \multicolumn{5}{|l|}{ Número de moirões } \\
\hline & Equações de melhor ajuste & $\mathrm{F}_{\mathrm{cal}}$ & $\mathrm{R}^{2}(\%)$ & Syx (ud) & Syx \% \\
\hline $\begin{array}{l}\text { Delfim } \\
\text { Moreira }\end{array}$ & $\operatorname{Ln}(N M)=-4,1213523873+0,7573355172 * \operatorname{Ln}\left(\left(D^{2} P^{2}\right) * H T\right)$ & 489,58 & 90,55 & $\pm 2,33$ & 29,43 \\
\hline Aiuruoca & $\operatorname{Ln}(N M)=-3,6638615174+0,7144828424 * \operatorname{Ln}\left(\left(D_{A P}^{2}\right) * H T\right)$ & 611,34 & 91,19 & $\pm 3,52$ & 33,54 \\
\hline Ouro Preto & $\operatorname{Ln}(N M)=-4,3479890317+0,7799878756 * \operatorname{Ln}\left(\left(D^{2} P^{2}\right) * H T\right)$ & 527,67 & 89,62 & $\pm 3,22$ & 31,77 \\
\hline
\end{tabular}

$\mathrm{F}_{\text {cal }}$ - valor de $\mathrm{F}$ calculado; $\mathrm{R}^{2}$ - coeficiente de determinação corrigido; Syx - erro padrão da estimativa; ud - unidade.

3.2 Quantificação do volume, fator de empilhamento e número de moirões médio por classe de diâmetro

Na Tabela 5, são mostrados os valores médios das árvores cubadas rigorosamente, juntamente com os valores do desvio padrão da média, para as variáveis estudadas. A distribuição diamétrica da candeia tem comportamento semelhante nos três municípios em estudo, ou seja, há um aumento contínuo e, normalmente, as árvores atingem até $32,5 \mathrm{~cm}$ de DAP, tendo sido encontrados exemplares com até $54,11 \mathrm{~cm}$ de DAP.

A altura das maiores árvores nos municípios de Delfim Moreira e Aiuruoca situa-se na faixa de 9,60 a 10,80 $\mathrm{m}$, mas foram encontrados indivíduos com até 12,20 m. Já, para o município de Ouro Preto, a altura das maiores árvores está entre 10,50 e $12,00 \mathrm{~m}$, tendo sido encontrados indivíduos com até $13,80 \mathrm{~m}$.

Verificando-se as relações entre os valores de volume total com casca para o município de Ouro Preto, constatou-se que as plantas com diâmetro na classe com valor central de 7,5 cm apresentam 3,07 vezes menos volume que aquelas com diâmetro na classe de $12,5 \mathrm{~cm}$; 7,52 vezes menos que aquelas com diâmetro na classe de
$17,5 \mathrm{~cm} ; 14,30$ vezes menos que aquelas com diâmetro na classe de $22,5 \mathrm{~cm} ; 24$ vezes menos que aquelas com diâmetro na classe de $27,5 \mathrm{~cm}$ e 34,38 vezes menos que aquelas com diâmetro na classe de $32,5 \mathrm{~cm}$.

Essas relações são importantes para o manejo florestal sustentável, quando for preciso decidir até que diâmetro é mais viável conduzir o povoamento, já que a medida que o diâmetro aumenta, é natural que a proporção entre esse e os demais diâmetros diminua (SCOLFORO \& THIERSCH, 2004).

A porcentagem de casca decresceu das menores para as maiores árvores, nos três municípios estudados. Segundo Scolforo \& Thiersch (2004), isto é o que normalmente ocorre nos plantios de eucalipto, pinus ou qualquer outra espécie florestal em que o espaçamento mais adensado faz com que a proporção entre o volume contido na copa em relação ao volume contido no fuste seja pequena.

O valor médio do fator de empilhamento é de 1,92 para as árvores avaliadas no município de Delfim Moreira, de 1,91 para Aiuruoca e de 1,98 para Ouro Preto. Segundo (SCOLFORO \& THIERSCH, 2004), o fator de empilhamento tende a decrescer com o aumento da classe de diâmetro, já que quanto maior o diâmetro das peças, mais madeira estará contida em um metro estéreo.

Cerne, Lavras, v. 16, n. 4, p. 431-441, out./dez. 2010 

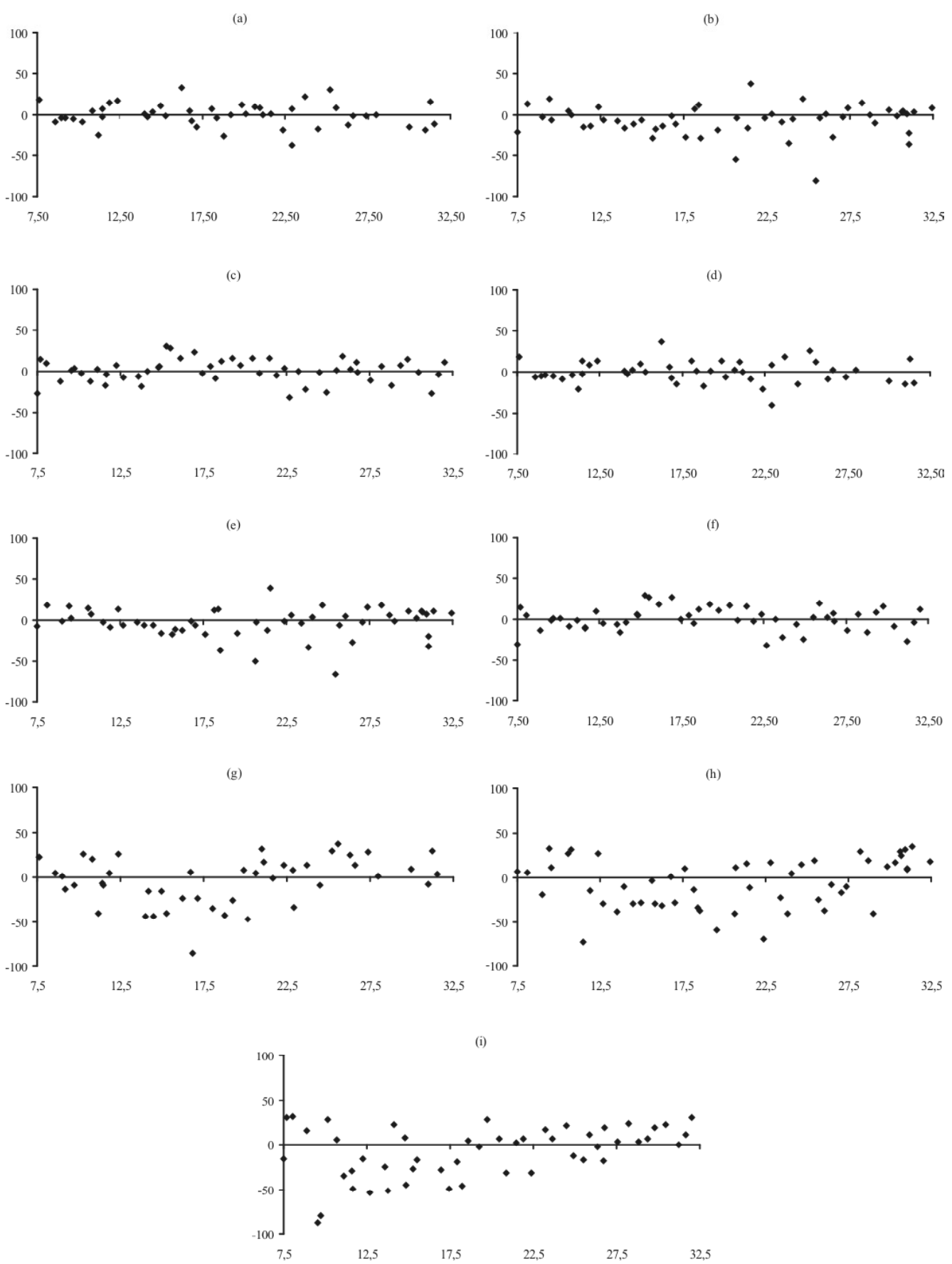

Figura 3 - Distribuição gráfica de resíduos para: volume total com casca das árvores cubadas no município de Delfim Moreira (a), Aiuruoca (b) e Ouro Preto (c); volume total sem casca para as árvores do município de Delfim Moreira (d), Aiuruoca (e) e Ouro Preto (f); número de moirões para as árvores dos municípios de Delfim Moreira (g), Aiuruoca (h) e Ouro Preto (i).

Figure 3 - Residual graphic distribution for: total volume with bark of the scaled trees from Delfim Moreira $(a)$, Aiuruoca $(b)$ and Ouro Preto (c) counties; total volume without bark for Delfim Moreira (d), Aiuruoca (e) and Ouro Preto (f) counties; number of fence posts for Delfim Moreira ( $g$ ), Aiuruoca $(h)$ and Ouro Preto (i) counties. 


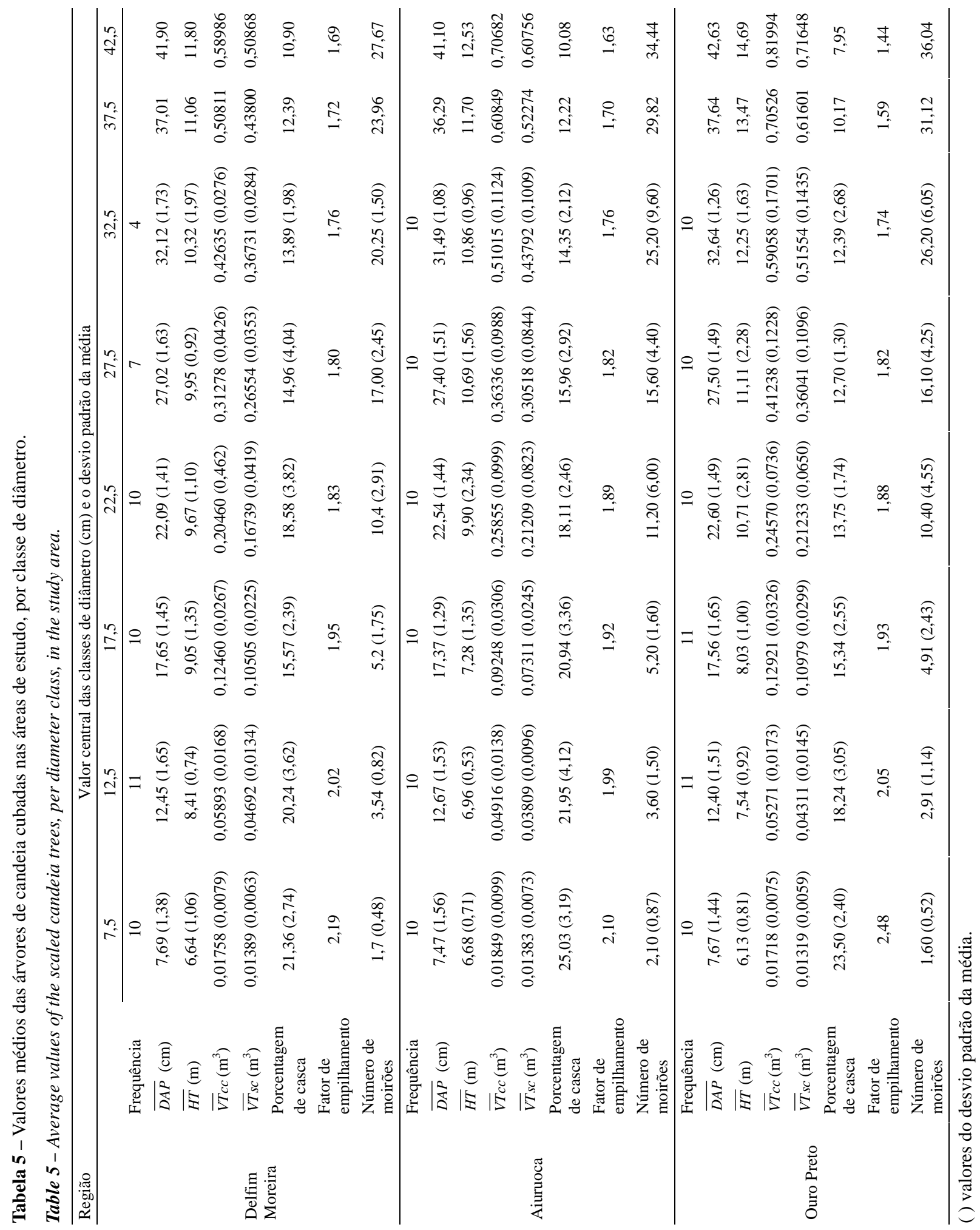

Cerne, Lavras, v. 16, n. 4, p. 431-441, out./dez. 2010 
Na Figura 4, mostram-se, de forma análoga, os dados da Tabela 5, para os três municípios estudados. Observase que, para as variáveis volume total com casca, volume total sem casca e número de moirões, a tendência é crescente em função do aumento da classe de diâmetro. Já, para o fator de empilhamento, o comportamento se apresenta decrescente, em função do aumento das classes de diâmetro.

\subsection{Teste de identidade entre modelos}

Na Tabela 6, são apresentados os resultados dos testes de identidade entre modelos ao nível de $5 \%$ de probabilidade. Para a variável volume total com casca obtiveram-se os seguintes resultados: os testes de identidade, nível e forma foram significativos para a combinação dos municípios de Delfim Moreira, Aiuruoca e Ouro Preto, e para a combinação Delfim Moreira e Ouro Preto; para a Aiuruoca e Ouro Preto
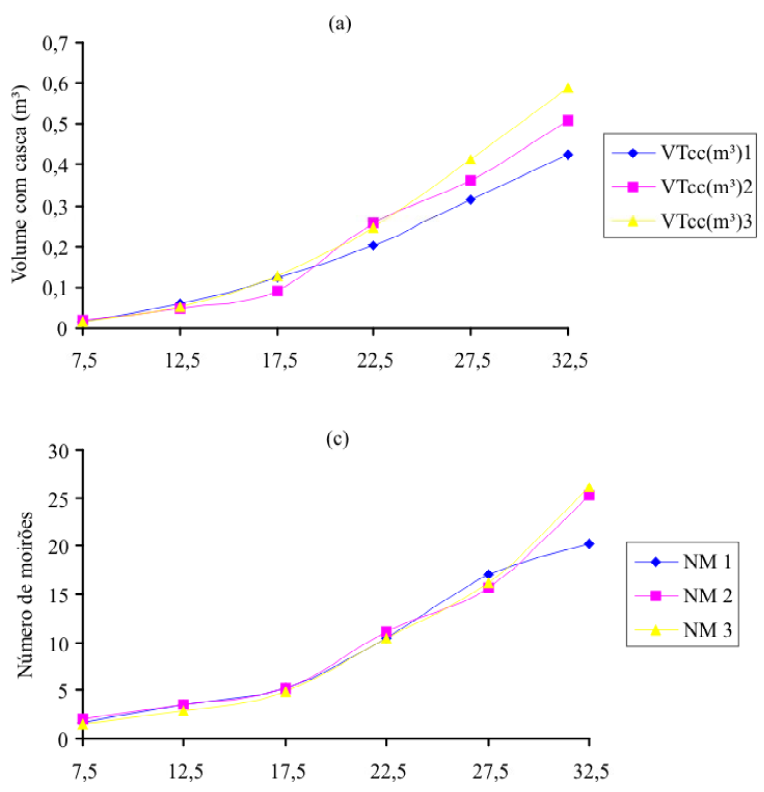

esses testes foram não significativos; para a combinação Delfim Moreira e Aiuruoca, o teste de identidade foi não significativo e, para nível e forma, foi significativo.

Assim, pode-se inferir que para os municípios de Aiuruoca e Ouro Preto as equações para o volume total com casca são semelhantes, podendo-se utilizar apenas uma equação para estimar essa variável (Tabela 7).

Em relação a variável volume total sem casca, também pode ser usada apenas uma equação para os municípios de Aiuruoca e Ouro Preto já que essa combinação apresentou resultados não significativos para os testes de identidade, nível e forma.

Para a variável número de moirões, em todas as combinações possíveis entre os três municípios, não houve a possibilidade de agrupamento. Então, não existe semelhança entre os municípios e deve ser utilizada uma equação para cada um deles.
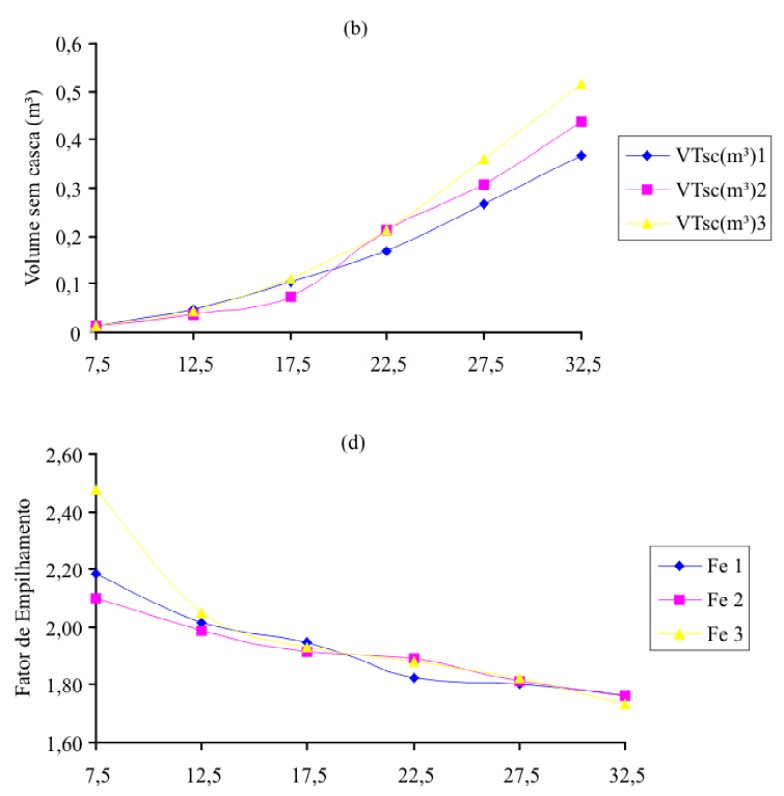

Figura 4 - Comportamento do volume total com casca (a), volume total sem casca (b), número de moirões (c) e fator de empilhamento (d), em relação à classe de diâmetro.

Figure 4-Tendency curves of the variables total volume with bark (a), total volume without bark (b), number of fence posts (c), and stack factor $(d)$. 
Tabela 6 - Resultado do teste de identidade, nível e forma, para as variáveis estudadas em todas as combinações entre os municípios de Delfim Moreira (1), Aiuruoca (2) e Ouro Preto (3).

Table 6 - Results from the identity test, level and form, for the studied variables considering all combinations among Delfim Moreira (1), Aiuruoca (2) and Ouro Preto (3) counties.

\begin{tabular}{|c|c|c|c|c|c|}
\hline Variável & Combinação & Teste & $\mathrm{F}_{\mathrm{cal}}$ & $\mathrm{F}_{\mathrm{tab}}$ & Teste \\
\hline \multirow{12}{*}{ Volume Total com casca } & 123 & Identidade & 3,95 & 2,42 & $\mathrm{~S}$ \\
\hline & 123 & Nível & 6,52 & 3,05 & S \\
\hline & 123 & Forma & 7,61 & 3,05 & $S$ \\
\hline & 23 & Identidade & 1,97 & 3,07 & NS \\
\hline & 23 & Nível & 2,34 & 3,92 & NS \\
\hline & 23 & Forma & 3,58 & 3,92 & NS \\
\hline & 13 & Identidade & 7,26 & 3,08 & $S$ \\
\hline & 13 & Nível & 12,88 & 3,93 & S \\
\hline & 13 & Forma & 14,52 & 3,93 & S \\
\hline & 12 & Identidade & 2,75 & 3,08 & NS \\
\hline & 12 & Nível & 5,41 & 3,93 & $\mathrm{~S}$ \\
\hline & 12 & Forma & 5,30 & 3,93 & S \\
\hline \multirow{12}{*}{ Volume Total sem casca } & 123 & Identidade & 3,68 & 2,42 & $S$ \\
\hline & 123 & Nível & 6,46 & 3,05 & $S$ \\
\hline & 123 & Forma & 7,28 & 3,05 & S \\
\hline & 23 & Identidade & 1,79 & 3,07 & NS \\
\hline & 23 & Nível & 2,79 & 3,92 & NS \\
\hline & 23 & Forma & 3,54 & 3,92 & NS \\
\hline & 13 & Identidade & 6,93 & 3,08 & $S$ \\
\hline & 13 & Nível & 12,64 & 3,93 & S \\
\hline & 13 & Forma & 13,83 & 3,93 & S \\
\hline & 12 & Identidade & 2,49 & 3,08 & NS \\
\hline & 12 & Nível & 4,69 & 3,93 & S \\
\hline & 12 & Forma & 4,94 & 3,93 & S \\
\hline \multirow{12}{*}{ Número de Moirões } & 123 & Identidade & 6,11 & 2,42 & $S$ \\
\hline & 123 & Nível & 6,50 & 3,05 & S \\
\hline & 123 & Forma & 5,85 & 3,05 & S \\
\hline & 23 & Identidade & 5,43 & 3,07 & $S$ \\
\hline & 23 & Nível & 2,20 & 3,92 & NS \\
\hline & 23 & Forma & 0,07 & 3,92 & NS \\
\hline & 13 & Identidade & 7,01 & 3,08 & $S$ \\
\hline & 13 & Nível & 6,50 & 3,93 & $S$ \\
\hline & 13 & Forma & 11,00 & 3,93 & S \\
\hline & 12 & Identidade & 6,85 & 3,08 & S \\
\hline & 12 & Nível & 13,23 & 3,93 & S \\
\hline & 12 & Forma & 10,36 & 3,93 & $\mathrm{~S}$ \\
\hline
\end{tabular}

Cerne, Lavras, v. 16, n. 4, p. 431-441, out./dez. 2010 
Tabela 7 - Equações selecionadas e medidas de precisão para os municípios estudados.

Table 7 - Selected equations and the accuracy measures for the studied counties.

\begin{tabular}{|c|c|c|c|c|c|}
\hline $\begin{array}{l}\text { Variável } \\
\text { dependente }\end{array}$ & Município(s) & Equação & $\begin{array}{l}\mathrm{R}_{\text {ajustado }}^{2} \\
(\%)\end{array}$ & $\begin{array}{l}\text { Syx } \\
\left(\mathrm{m}^{3}\right)\end{array}$ & Syx $(\%)$ \\
\hline \multirow{2}{*}{$\begin{array}{l}\text { Volume total } \\
\text { com casca }\end{array}$} & $\begin{array}{l}\text { Aiuruoca } \\
\text { Ouro Preto }\end{array}$ & $L n V T_{c c}=-10,0663618557+1,0081812391 * \operatorname{Ln}\left(\left(D A P^{2}\right) * H T\right)$ & 98,26 & $\pm 0,05567$ & 24,62 \\
\hline & D. Moreira & $L n V T_{c c}=-10,0695707796+1,002283589^{*} \operatorname{Ln}\left(\left(D A P^{2}\right)^{*} H T\right)$ & 98,45 & $\pm 0,0308$ & 19,97 \\
\hline \multirow{2}{*}{$\begin{array}{l}\text { Volume total } \\
\text { sem casca }\end{array}$} & $\begin{array}{c}\text { Aiuruoca } \\
\text { Ouro Preto }\end{array}$ & $L n V T_{s c}=-10,5504605249+1,0445453331 * \operatorname{Ln}\left(\left(D A P^{2}\right) * H T\right)$ & 98,29 & $\pm 0,04761$ & 24,72 \\
\hline & D. Moreira & $L n V T_{s c}=-10,471757251+1,0284915738 * \operatorname{Ln}\left(\left(D A P^{2}\right) * H T\right)$ & 98,60 & $\pm 0,0240$ & 18,62 \\
\hline \multirow{3}{*}{$\begin{array}{l}\text { Número de } \\
\text { moirões }\end{array}$} & D. Moreira & $\operatorname{LnNM}=-4,1213523873+0,7573355172 * \operatorname{Ln}\left(\left(D A P^{2}\right) * H T\right)$ & 90,55 & $\pm 2,3300$ & 29,43 \\
\hline & Aiuruoca & $\operatorname{LnNM}=-3,6638615174+0,7144828424 * \operatorname{Ln}\left(\left(D A P^{2}\right)^{*} H T\right)$ & 91,19 & $\pm 3,5200$ & 33,54 \\
\hline & Ouro Preto & $\operatorname{LnNM}=-4,3479890317+0,7799878756 * \operatorname{Ln}\left(\left(D A P^{2}\right) * H T\right)$ & 89,62 & $\pm 3,2200$ & 31,77 \\
\hline
\end{tabular}

\section{CONCLUSÕES}

O fator de empilhamento decresce com o aumento das classes diamétricas.

O modelo selecionado para todas as variáveis testadas foi o de Spurr logaritmizado.

$\mathrm{O}$ teste de identidade entre modelos indicou a possibilidade de agrupamento entre os municípios de Aiuruoca e Ouro Preto para as variáveis volume total com casca e volume total sem casca, enquanto que para a variável número de moirões esse teste indicou não ser possível o agrupamento de nenhuma combinação entre os municípios estudados.

\section{REFERÊNCIAS BIBLIOGRÁFICAS}

CARVALHO, P. E. R. Espécies florestais brasileiras: recomendações silviculturais, potencialidade e uso da madeira. Brasília: Embrapa, 1994. 640 p.
GRAYBILL, F. A. Theory and application of the linear model. Belmont: Duxbury, 1976.

PÉREZ, J. F. M. Sistema de manejo para a candeia (Eremanthus erythropappus (DC) MacLeish). 2001. 71 p. Dissertação (Mestrado em Engenharia Florestal) - Universidade Federal de Lavras, Lavras, 2001.

SCOLFORO, J. R. S. Biometria florestal: parte I, modelos de regressão linear e não linear: parte II, modelos para relação hipsométrica, volume, afilamento e peso de matéria seca. Lavras: UFLA, 2005. $351 \mathrm{p}$.

SCOLFORO, J. R. S.; THIERSCH, C. R. Biometria florestal: medição, volumetria e gravimétrica. Lavras: UFLA/FAEPE, 2004. $285 \mathrm{p}$. 
